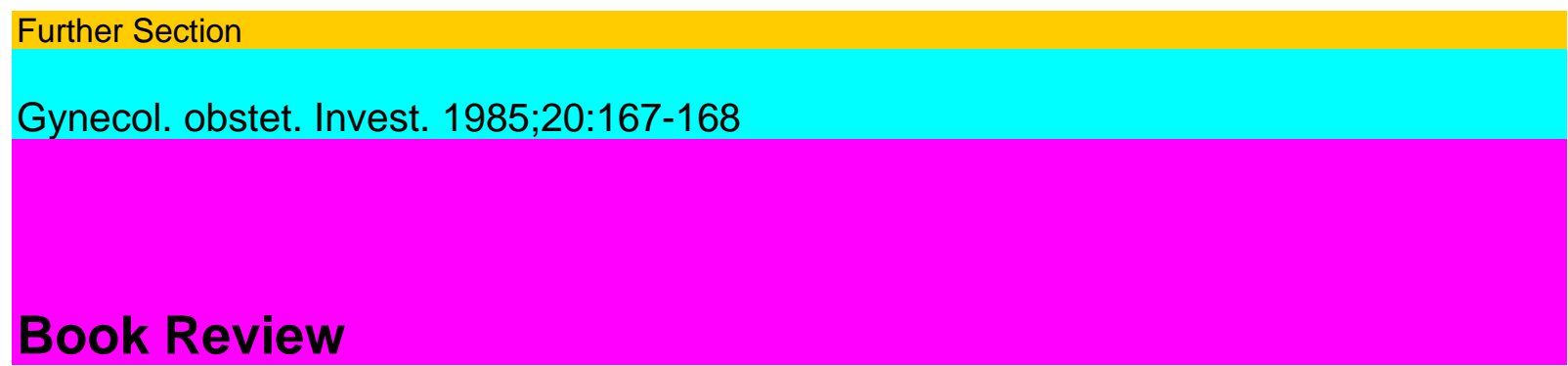

\title{
H. Schönfeld
}

Prevention of Perioperative Infections

Antibiotics and Chemotherapy, vol. 33

Karger, Basel 1985

VIII + 208 pp.; SFr. 149.-/DM 178.-/

US\$ 89.25

ISBN 3-8055-3936-3

This volume consists of nine presentations related to the topic of perioperative infections. As with any multiauthored production, the content as well as the literary effort varies, and unfortunately the weakest of all the contributions is that pertaining to hysterectomy.

The introductory chapter pertaining to genitourinary surgery is well written and gives one a great deal of food for thought. What is relevant to all clinicians is that there are high rates of bacteriuria following cath-eterization, and as indicated in this chapter 5-16\% of patients in community hospitals have Foley catheters. It is estimated that approximately 1 million nosocomial urinary tract infections occur annually as a result of instrumentation and catheterization in the United States. The author emphasizes that although many cases of catheter- and instrumentassociated bacteriuria are asymptomatic, at least 1-2 \% are associated with secondary bacteremia, and the fatality rate for nosocomial urinary tract infection is $1 \%$. There is no doubt that patients are better having infection with their own Escherichia coli than those strains distributed by hospital staff who do not obey the rules for scrupulous catheter care. The author emphasizes that only those persons, be they hospital personnel, family members or patients, who are familiar with and have been thoroughly checked out on the procedure of aseptic catheterization and maintenance, should handle catheters. It is also a sound recommendation that patients should be given periodic inservice training and be apprised of the complications associated with urinary catheterization.

The following chapter on perioperative prevention of infection in abdominal surgery is also well written and contains a great deal of useful information. As is well known, prolonged preoperative hospitalization

increases wound infection, and aseptic and antiseptic techniques cannot overcome poor surgical technique.

The presentation on the prevention of infection in high-risk biliary operations is also a lucid review and brings out the well-know point that it is difficult to determine the incidence of highrisk factors in the clinical practice of certain surgeons who claim that they see no sepsis! The following remark is germane: 'It has ever been true that there are none so blind as will not see'. Similarly, the quotation pertaining to prospective audits and retrospective studies is also pertinent: 'The retrospectivists are experts of the art of selective forgetfulness'.

Other contributors emphasize that there is absolutely no doubt that the most important single variable in the prevention of postoperative septic complications is the expertise of the individual surgeon. 
The contribution on perioperative antibiotics for hysterectomy is an abysmal literary effort.

There are sentences which one would not expect from someone whose mother tongue is English! One realizes that the surgery in the author's hospital is often performed by residents who may be poorly supervised, which explains the high rate of postoperative sepsis. On the other hand, one also notes that the antibiotic trials which are being conducted resemble Ariadene's web.

Numerous aspects of perioperative antibiotics must be considered, including cost and changes in the nosocomial type of infections. The latter have not been addressed by the author. Since mechanisms of resistance already exist prior to widespread clinical use of the new beta-lactam antibiotics, it can be anticipated that the therapeutic life span of these compounds will be limited. The boundaries of these limits will be established by local use and abuse patterns.

The other contributions also emphasize that of the many antimicrobials currently available for the treatment of gram-negative and gram-positive infections none has a spectrum which includes a majority of likely pathogens. The ever increasing choice among the range of antibiotics and the array of diverse sources of drug information means that rational informed prescribing habits must currently be difficult. Confusion and lack of knowledge of antibiotic therapy are well known. More and more numerous antibiotics

168

Book Review

appear each year, and only rarely are they beneficial when compared with the congeners. Lack of information has frequently been responsible for unexplained therapeutic failures or complications including an increase in nosocomial infections. Such complications and failure may occur at different levels and lack of knowledge on the part of the physician may result in striking iatrogenic consequences. They result either from competition for binding to plasma albumin or from induction or inhibition of hepatic enzymes responsible for biotransformation. In other words, physicians should be aware of the multiple dangers of polypharmacy. This volume does not help in this respect because it forgets that what is new is not always the best either for prophylaxis or treatment.

Finally, the cost of this volume is prohibitive and it will only find its place in libraries, but there are chapters which should be read by all who are involved with surgical subspecialties.

David Charles, Huntington, W.Va. 